\title{
Young's modulus and residual stress of GeSbTe phase-change thin films
}

\author{
Hammad Nazeer ${ }^{\mathrm{a}}$, Harish Bhaskaran ${ }^{\mathrm{b}}$, Léon A Woldering ${ }^{\mathrm{a}}$, Leon \\ Abelmann $^{\mathrm{a}, \mathrm{c}, *}$ \\ ${ }^{a} \mathrm{MESA}^{+}$Research Institute, University of Twente \\ ${ }^{b}$ Department of Materials, University of Oxford, Oxford, United Kingdom \\ ${ }^{c}$ KIST Europe, Saarbrücken, Germany
}

\begin{abstract}
The mechanical properties of phase change materials alter when the phase is transformed. In this paper, we report on experiments that determine the change in crucial parameters such as Young's modulus and residual stress for two of the most widely employed compositions of phase change films, $\mathrm{Ge}_{1} \mathrm{Sb}_{2} \mathrm{Te}_{4}$ and $\mathrm{Ge}_{2} \mathrm{Sb}_{2} \mathrm{Te}_{5}$, using an accurate microcantilever methodology. The results support understanding of the exact mechanisms that account for the phase transition, especially with regards to stress, which leads to drift in non-volatile data storage. Moreover, detailed information on the change in mechanical properties will enable the design of novel low-power nonvolatile MEMS.

Keywords: Youngs modulus, strain, phase change, GeSbTe, cantilever resonance
\end{abstract}

\section{Introduction}

The present quest in Nanoelectromechanical Systems (NEMS) and Microelectromechanical Systems (MEMS) is for ever-lighter and lower power technologies, thus there is a constant effort to reduce device dimensions. The use 5 of phase change materials in such devices is a rather nascent concept, and is stymied by the lack of understanding of the exact nature of the mechanical

\footnotetext{
${ }^{*}$ Corresponding author

Email address: +49 6819382229, 1.abelmann@utwente.nl (Leon Abelmann)
}

Preprint submitted to Thin Solid Films

September 7, 2015 
property change in such materials. However, an understanding of the phase change transition will be transformational. For example, tunable resonators all require high speed tuning of the resonance frequency of mechanical resonators. This is mostly achieved using electrostatic tuning, which has other issues such as the requirement to maintain a voltage, electrostatic spring softening, which competes with resonator stiffening due to tension in beam structures as well as exacting impedance matching requirements for high-speed operation. The modulation of a resonator material's Young's modulus is the most direct way to affect the resonator's frequency. This has been used in the reverse to detect magnetic fields [1, 2, 3, whereby the the magnetostriction in films is modulated by magnetic fields, which in turns changes the Young's Modulus of the microcantilever. However, the use of phase change materials in such devices could be potentially transformational, as these materials retain the phase state (amorphous/crystalline ratio) for many decades, thus requires infrequent tuning, but remain capable of a broad range depending on the change in modulus.

Phase change materials have also formed the dominant non-magnetic data storage medium (from rewritable DVDs to Blu-Ray Disks) of the last couple of decades, and more recently in the form of the highly scalable phase change memories. In the context of the latter, where multiple bits are stored in a single cell, based on changes in resistivity, the stress induced by the phase change itself could result in resistance drift [4. However, a clear understanding of these issues in such materials is strikingly absent. In the context of emerging optoelectronics applications of these materials [5, 6, 7], such an understanding would be vital to be able to design for variation induced by the change in the mechanical properties of these materials. This is especially so when coupled to elements mismatched in thermal expansion coefficient, such as silicon-nitride.

Mechanical properties of thin films, notably their Young's modulus, can be accurately determined by means of microcantilevers [8, 9, 10, 11, 12, 13, 35 14. The method is particularly usefull if the available substrate area is in the millimeter range, for instance due to small deposition area (such as in pulsed laser deposition) or because the film has variations in mechanical properties on 
the millimeter range. In these situations, methods like wafer curvature or X-ray diffraction are difficult to apply, and less accurate.

The microcantilever method relies on a change in resonance frequency caused by the deposition of the thin film on the cantilever. The extra mass of the thin film will decrease the resonance frequency, whereas the added rigidity of the structure by adding the film will cause an increase. Up to now, thin films from different deposition runs have been investigated, for instance to determine the variation of the Young's modulus with film composition [14. In this paper we illustrate the method on GeSbTe (GST) phase change films, which has the advantage that the Young's modulus of the film can be changed when it is on the cantilever, without changing its mass. The sole reason for a change in resonance frequency will therefore be the change in the Young's modulus of the phase change film.

Phase change materials based on the Ge-Sb-Te (GST) alloy are found to exhibit excellent electrical and phase change properties and can endure large numbers of read write cycles [15. From the family of GST alloys, the compositions $\mathrm{Ge}_{1} \mathrm{Sb}_{2} \mathrm{Te}_{4}$ (GST124) and $\mathrm{Ge}_{2} \mathrm{Sb}_{2} \mathrm{Te}_{5}$ (GST225) are widely studied because of their superior combination of properties [16, 17, 18. These materials were mostly investigated on the basis of their optical, electrical and phase change properties.

To support the use of these materials as an active device layer, information is needed on the mechanical properties, like Young's modulus and residual stress. ${ }_{60}$ These properties have not been extensively investigated and a large variation of reported values can be found in literature [19, 20. Knowledge on the compositional dependence of these properties and their variation with temperature facilitates the choice for the correct composition for a particular application.

In this paper we report on accurate measurements of Young's modululs and 65 residual stresses on technologically relevant thin films of phase change materials. The theoretical relations for the properties investigated in this study are explained in section 2 Cantilevers were fabricated with a standard MEMS fabrication process and GST thin films were sputtered on these cantilevers (see 

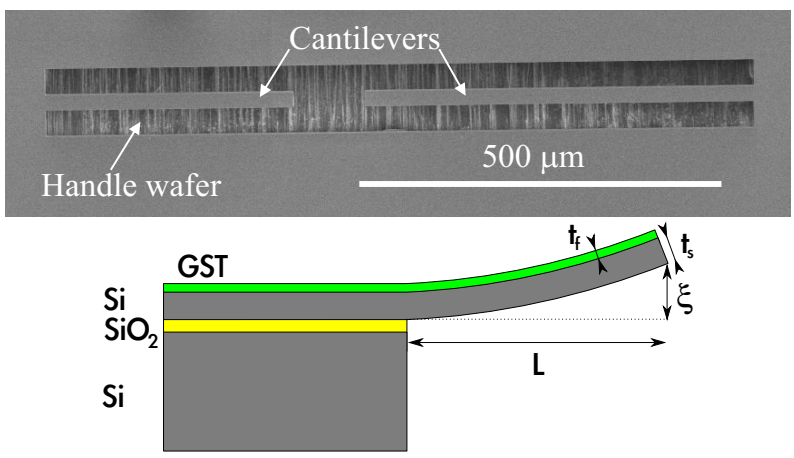

Figure 1: Top: Scanning electron micrograph of cantilevers used, fabricated from a $3 \mu \mathrm{m}$ thick silicon device layer. Their length varies from $250 \mu \mathrm{m}$ to $350 \mu \mathrm{m}$ in steps of $10 \mu \mathrm{m}$. The cantilevers have a constant width of $30 \mu \mathrm{m}$. Bottom: Model used for calculation.

section 3). In this section, we explain the annealing treatment and measurement procedures as well. In addition to the determination of the Young's modulus, we determined the residual stress of the GST thin films from the change in static deflection of the cantilevers. Since crystallization of the GST thin films is accompanied by a volume reduction [21, it results in an abrupt change in the cantilever deflection. The mechanical measurements are complemented by electrical characterisation and discussed in section 4

\section{Theory}

The resonance frequency shift and bending due to crystallisation of the phase-change material can be calculated by assuming a simple, single sided clamped cantilever (Figure1).

2.1. Analytical model for the Young's modulus of GST thin films in amorphous and crystalline states

A dynamic approach, based on the Euler-Bernoulli beam equation and the dependence of the resonance frequency on the flexural rigidity of composite cantilever, was used to develop an analytical model for determination of the

${ }_{85}$ Young's modulus [11. In contrast to the work of Won et al, no approximation has been made 13 . 
Due to the addition of a thin film on the cantilever, the flexural rigidity of the composite cantilever increases and its neutral plane (zero strain) shifts. In addition the film will add to the mass of the composite cantilever. These three effects result in a change in the resonance frequency. By measuring this change before and after deposition of the thin film, and before and after crystallisation, one can obtain the variation in Young's modulus from equation 1.

$$
\begin{aligned}
& E_{\mathrm{f}}^{*}=\frac{1}{t_{\mathrm{f}}^{3}}\left[6\left(\gamma_{\mathrm{s}}+\gamma_{\mathrm{f}}\right) B-2 E_{\mathrm{s}}^{*} t_{\mathrm{s}}^{3}-3 t_{\mathrm{f}} E_{\mathrm{s}}^{*} t_{\mathrm{s}}^{2}-2 E_{\mathrm{s}}^{*} t_{\mathrm{s}} t_{\mathrm{f}}^{2}\right. \\
& \left.+2 \sqrt[\begin{array}{l}
E_{\mathrm{s}}^{* 2} t_{\mathrm{s}}^{2} t_{\mathrm{f}}^{4}+3 E_{\mathrm{s}}^{* 2} t_{\mathrm{s}}^{3} t_{\mathrm{f}}^{3}+\left(4 E_{\mathrm{s}}^{* 2} t_{\mathrm{s}}^{4}-3 A B\right) t_{\mathrm{f}}^{2}+ \\
\left(3 E_{\mathrm{s}}^{* 2} t_{\mathrm{s}}^{5}-9 A B t_{\mathrm{s}}\right) t_{\mathrm{f}}+E_{\mathrm{s}}^{* 2} t_{\mathrm{s}}^{6}-6 A B t_{\mathrm{s}}^{2}+ \\
9\left(\gamma_{\mathrm{s}}+\gamma_{\mathrm{f}}\right)^{2} B^{2}
\end{array}],\right]{ }
\end{aligned}
$$

where

$$
A=E_{\mathrm{s}}^{*} t_{\mathrm{s}}\left(\gamma_{\mathrm{s}}+\gamma_{\mathrm{f}}\right)
$$

and

$$
B=\left(\sqrt{\frac{E_{\mathrm{s}}^{*} t_{\mathrm{s}}^{3}}{12 t_{\mathrm{s}} \rho_{\mathrm{s}}}}-0.568 \pi \Delta f_{0} L^{2}\right)^{2} .
$$

In the above equation $L, t$ and $E^{*}$ are the length [m], thickness [m] and the effective Young's modulus $[\mathrm{Pa}]$ respectively. The effective density $\gamma=\rho t$ $\left[\mathrm{kg} / \mathrm{m}^{2}\right]$. Subscripts 's' and 'f' denote the silicon and the thin film. The measured difference in the fundamental resonance frequency of the cantilevers before and after the deposition of the thin film is denoted by $\Delta f_{0}$. By taking this difference, any potential uncertainties in the thickness of the cantilever can be eliminated and a more accurate result is obtained [22].

The resonance frequency of the cantilever is a function of the Young's modulus of the GST film and its mass, which is the product of density, density and area. We assume the mass and area of the film do not change during a transition from the amorphous to crystalline phase. The change in resonance frequency therefore is in first order only caused by a change in Young's modulus. Due 
to crystalisation however, the density increases, resulting in a decrease in film thickness and a shift of the neutral plane towards the cantilever axis. This will induce a resonance frequency shift by itself. Fortunately, this is a negligible effect. From equation 1 we calculate that for a $200 \mathrm{~nm}$ GST film deposited on a $3 \mu \mathrm{m}$ silicon cantilever, the product of Young's modulus and film thickness only changes by $0.3 \%$ when reducing the film thickness by $6.5 \%$. Therefore, we consider the product of GST Young's modulus and film thickness to be independent on a reduction in film thickness due to the crystallization process.

\subsection{Residual stress}

Crystallization of the GST thin films at elevated temperature leads to a reduction in volume. When deposited on cantilevers, this reduction leads to stress which causes the cantilevers to bend upwards. By measuring the static deflection of these cantilevers before and after the deposition at every annealing step, we can determine the residual stress in GST thin films at different temperatures. Since the GST film thickness is small compared to the substrate thickness, we can use Stoney's approximation [23, 24].

$$
\sigma_{\mathrm{f}}=\frac{1}{3} \frac{E_{\mathrm{s}} t_{\mathrm{s}}^{2} \xi}{\left(1-\nu_{\mathrm{s}}\right) t_{\mathrm{f}} L^{2}},
$$

the symbols $\sigma, E, \nu, t, L$, and $\xi$ are the residual stress, Young's modulus, Poison ratio, thickness, length and deflection respectively. Subscripts 's' and 'f' denote the silicon and thin film. Again, the product of residual stress and film thickness is independent on the film thickness itself.

\section{Experimental details}

\subsection{Fabrication of cantilevers}

The cantilever fabrication process given in the appendix. It is an improved version of the process previously reported in [11. The main differences are that we used a different recipe for the back side etch, which enables us to use foil on the front side instead of polyimide pyralin. After etching, the foil on the front 
side and photoresist material from the back side of the wafer were removed using $\mathrm{O}_{2}$ plasma (Tepla300E). Subsequently, the cantilevers were released by etching the BOX layer using buffered-hydrofluoric acid.

Scanning electron micrographs (SEM) as shown in figure 1 were used to inspect and characterise the fabricated cantilevers.

\subsection{GST deposition}

The $200 \mathrm{~nm}$ films of compositions $\mathrm{Ge}_{1} \mathrm{Sb}_{2} \mathrm{Te}_{4}$ (GST124) and $\mathrm{Ge}_{2} \mathrm{Sb}_{2} \mathrm{Te}_{5}$

140 (GST225) were deposited directly on the Si cantilevers by DC magnetron sputtering in an argon plasma at a sputtering power of $300 \mathrm{~W}$ and deposition rate of $6 \mathrm{~nm} / \mathrm{s}$. A $5 \mathrm{~nm} \mathrm{ZnS-SiO} 2$ capping layer was deposited to protect the films from oxidation at a sputtering power of $1 \mathrm{~kW}$ at a rate of $3.4 \mathrm{~nm} / \mathrm{s}$. Measurement of film thicknesses on microcantilevers is difficult and inaccurate. Therefore films thicknesses are estimated from deposition rates, calibrated by low angle x-ray diffraction and TEM measurements.

\subsection{Annealing of GST}

Compositional and phase dependence of the Young's modulus, residual stress and sheet resistance of the GST225 and GST124 thin films were investigated at different temperatures. Samples were annealed from room temperature to the desired temperature with a ramping rate of $3^{\circ} \mathrm{C} / \mathrm{min}$ in a vacuum furnace with nitrogen environment at a pressure of 1 mbar. The chips were kept at the desired temperature for 15 minutes and then cooled down to room temperature. The cooling rate was not controlled, but much lower than the heating rate.

Measurements for the Young's modulus, residual stress and sheet resistance were conducted at room temperature after each annealing step. Samples of different composition, identified by GST225 and GST124, were passed through an identical annealing procedure up to $180^{\circ} \mathrm{C}$ with reduced temperature steps around $150^{\circ} \mathrm{C}$. In order to investigate the behaviour of the properties around the crystallization temperature of GST124, a third sample with a $\mathrm{Ge}_{1} \mathrm{Sb}_{2} \mathrm{Te}_{4}$ composition (which we will refer to as GST124*) was annealed with extra temper- 
ature steps around $130^{\circ} \mathrm{C}$. The annealing history of the samples is summarized in table 1.

It should be noted that the cantilever fabrication process includes temperatures in excess of $350^{\circ} \mathrm{C}$, far above the temperatures used for the annealing of the GST films. Changes in cantilever resonance frequency and bending can therefore solely be attributed to the changes in the GST films.

Table 1: Annealing history of the GST thin films. GST124* passed through more annealing steps before reaching $140^{\circ} \mathrm{C}$.

\begin{tabular}{|c|c|c|c|c|c|c|c|c|c|c|}
\hline \multirow[b]{2}{*}{ Sample } & \multicolumn{10}{|c|}{ Annealing temperature $\left({ }^{\circ} \mathrm{C}\right)$} \\
\hline & 60 & 100 & 110 & 120 & 130 & 140 & 150 & 160 & 170 & 180 \\
\hline GST225 & $\mathrm{x}$ & $\mathrm{x}$ & & & & $\mathrm{x}$ & $\mathrm{x}$ & $\mathrm{x}$ & $\mathrm{x}$ & $\mathrm{x}$ \\
\hline GST124 & $\mathrm{x}$ & $\mathrm{x}$ & & & & $\mathrm{x}$ & $\mathrm{x}$ & $\mathrm{x}$ & $\mathrm{x}$ & $\mathrm{x}$ \\
\hline GST124* & $\mathrm{x}$ & $\mathrm{x}$ & $\mathrm{x}$ & $\mathrm{x}$ & $\mathrm{x}$ & $\mathrm{x}$ & $\mathrm{x}$ & $\mathrm{x}$ & $\mathrm{x}$ & $\mathrm{x}$ \\
\hline
\end{tabular}

\subsection{Resonance frequency measurements}

The resonance frequency of the cantilevers was measured by using a MSA400 micro system analyzer scanning laser Doppler vibrometer. Measurements of the resonance frequency were conducted both before and after the deposition and each annealing step. The thermal vibration at room temperature of the cantilevers in ambient conditions was used to measure the amplitude spectrum, which is then fitted to the theoretical expression of mass-spring system with damping to estimate the resonance frequency.

\subsection{Static deflection measurements}

Static deflection $\xi$ of the cantilevers was measured at room temperature by using white light interferometry (WLIF) of a Polytec MSA-400 analyzer. From the deflection, the residual stress in the GST thin films was determined using equation 2. As expected, all cantilevers were found to be straight before deposition of the GST thin films. After annealing, all cantilevers bent upwards, 


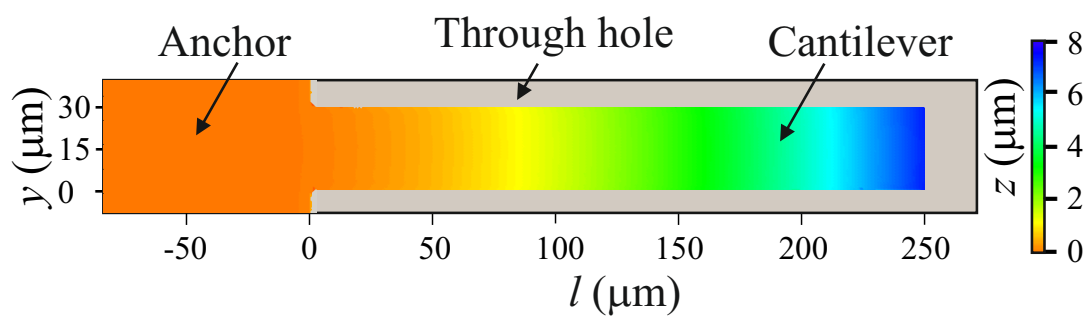

Figure 2: Top view of the reconstructed image from a white light interference measurement of an approximately $290 \mu \mathrm{m}$ long, $30 \mu \mathrm{m}$ wide and $3 \mu \mathrm{m}$ thick silicon cantilever, with $200 \mathrm{~nm}$ GST225 thin film, after annealing at $150^{\circ} \mathrm{C}$.

indicating that the GST films develop a tensile stress. A 2-D white light interference microscopic image of a $250 \mu \mathrm{m}$ long cantilever with GST225 after annealing at $150^{\circ} \mathrm{C}$ is shown in figure 2. From this image, the profile of the cantilever is obtained by averaging over the width. Cantilevers of varying length were measured, to reduce the error in the measurement due to uncertainty in the location of the cantilever base. All static deflection measurements were conducted at room temperature.

\subsection{Sheet resistance measurements}

The sheet resistance of the GST thin films was measured by a four-point probe on the handle wafer. The measurements were taken after the deposition of the GST thin films and after each annealing step at room temperature.

\section{Results and Discussion}

After deposition of the GST layers, the resonance frequencies of the cantilevers decrease by about $2 \mathrm{kHz}$ and they remain essentially straight. Since after deposition the GST layers are amorphous, they can be easily changed to the crystalline state by annealing in a standard oven. After anneal, the resonance frequency increases by about $500 \mathrm{~Hz}$ (figure 3), and the cantilevers bend upwards. Figure 4 shows that the end of a $250 \mu \mathrm{m}$ long cantilever bends up as much as $6 \mu \mathrm{m}$. These considerable effects can be used to accurately deter- 
mine the changes in Youngs modulus, stress and strain of the different GST compositions.

\subsection{Young's modulus}

From the shift in resonance frequency, the in-plane Young's modulus can be determined (see section 2). The Young modules of the GST thin films is shown in figure 5 as a function of composition and annealing step. In the amorphous state, below the crystallization temperature, the Young's modulus is lower than that of the corresponding crystalline phase [25].

During crystallization the thickness of the GST reduces, leading to an equal increase in film density. Since the mass and area of the film remain constant, the product of thickness and density will remains constant as well. The same is true, to a good approximation, for the product of Young's modulus and thickness. To be able to give absolute values, we have assumed the thickness reduction to be $6.5 \%$ for the GST225 composition [26, 27] (leading to an increase in density from $5870 \pm 50 \mathrm{~kg} / \mathrm{m}^{3}$ to $6270 \pm 20 \mathrm{~kg} / \mathrm{m}^{3}$ ), and a reduction of $4 \%$ for GST124 [28] (with density increasing from $5900 \mathrm{~kg} / \mathrm{m}^{3}$ to $6200 \mathrm{~kg} / \mathrm{m}^{3}$ ).

The measured Young's modulus of the GST225 thin film as deposited in amorphous state was found to be $18.9 \mathrm{GPa}$ with a standard error of $0.7 \mathrm{GPa}$ (indicate in the following by (0.7)). The Young's modulus increased sharply above the crystallization temperature of $150^{\circ} \mathrm{C}$ to a value of $38.2(0.3) \mathrm{GPa}$. The crystallization temperature agrees well with the range of values quoted in literature [16, 26, 29, 30]. The increase in the Young's modulus from amorphous to crystalline state is consistent with the results published in literature [31, 32 , 13. We assumed a typical value of $\nu_{\mathrm{f}}$ of 0.3 , in order to compare with the biaxial modulus values published in literature.

The Young's modulus of the GST124 thin film was found to be 15.9 (0.2) GPa and $31.3(0.3) \mathrm{GPa}$ in the amorphous and crystalline state respectively. The crystallization temperature of $130^{\circ} \mathrm{C}$ is consistent with the range of temperature values reported elsewhere [28, 33]. The fact that the crystallization temperature of GST124 is lower than GST225 agrees with measurements by Car- 

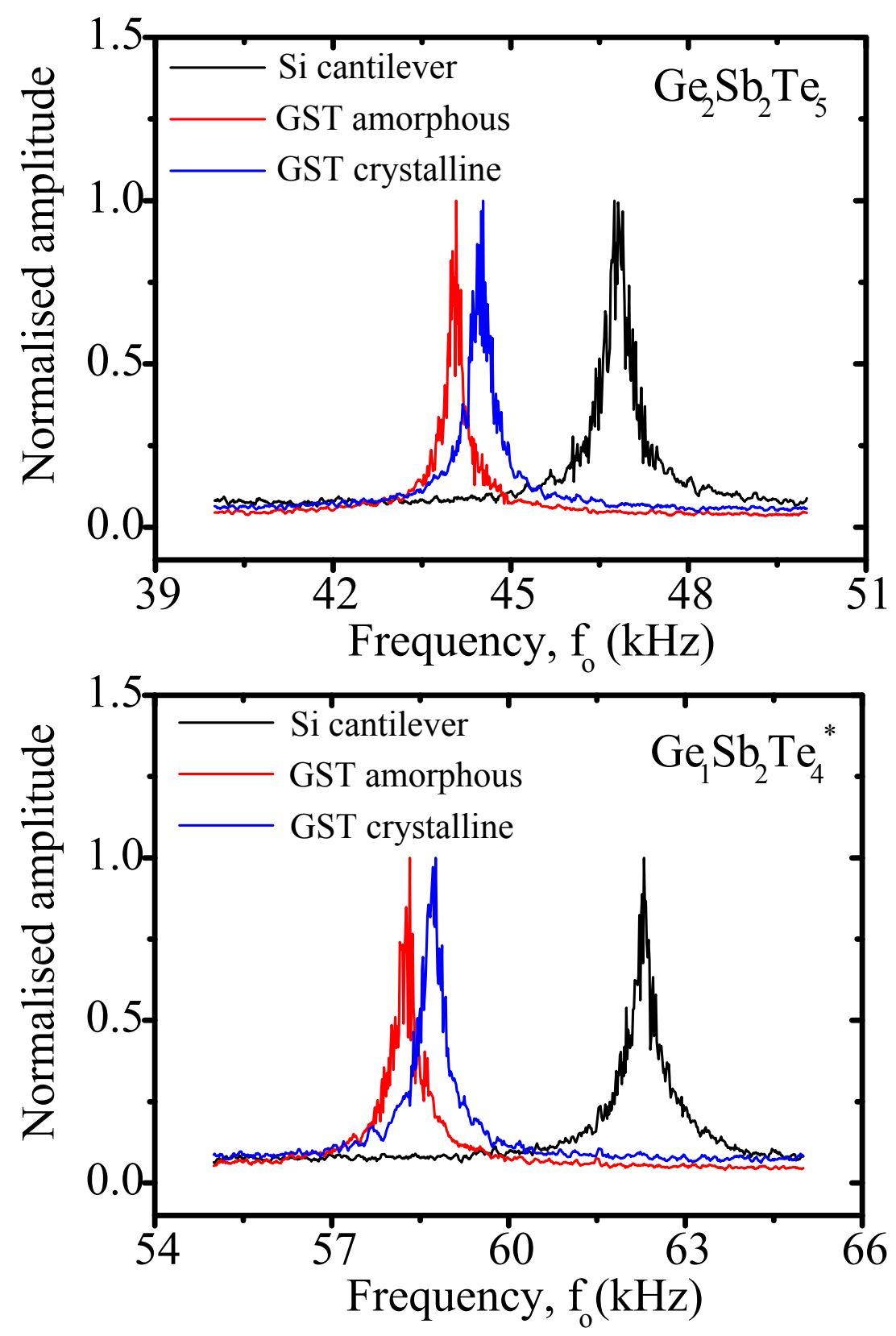

Figure 3: Measured resonance frequencies of the silicon cantilever without (black) and with GST thin films in amorphous (red) and crystalline state (blue). The resonance frequency decreases after deposition, whereas it increases upon annealing. This increase is attributed to the higher Young's modulus of the GST thin films in crystalline state. Top: ( $\mathrm{Ge}_{2} \mathrm{Sb}_{2} \mathrm{Te}_{5}$ on $\mathrm{a} \sim 310 \mu \mathrm{m}$ long cantilever. Bottom: $\mathrm{Ge}_{1} \mathrm{Sb}_{2} \mathrm{Te}_{4}$ on $\mathrm{a} \sim 260 \mu \mathrm{m}$ long cantilever. 


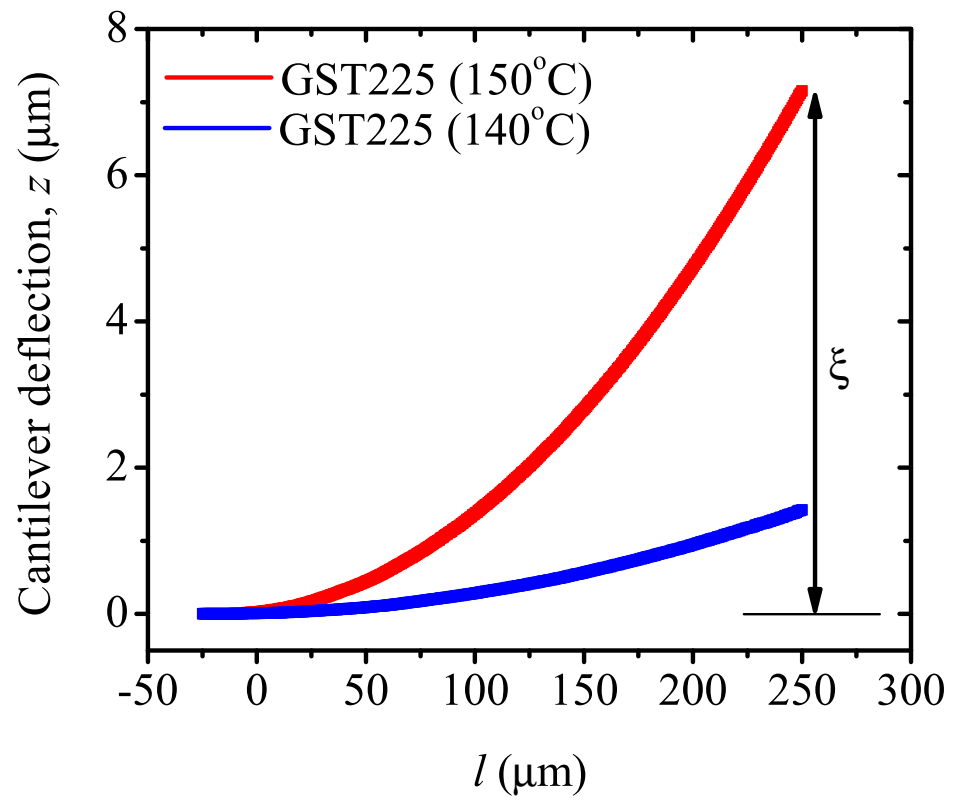

Figure 4: Cantilever profile after annealing, obtained from white light interferometry (figure 2. The measurements are taken at room temperature. The maximum upward static deflection increases with annealing temperature because of the built up of tensile residual stress. 


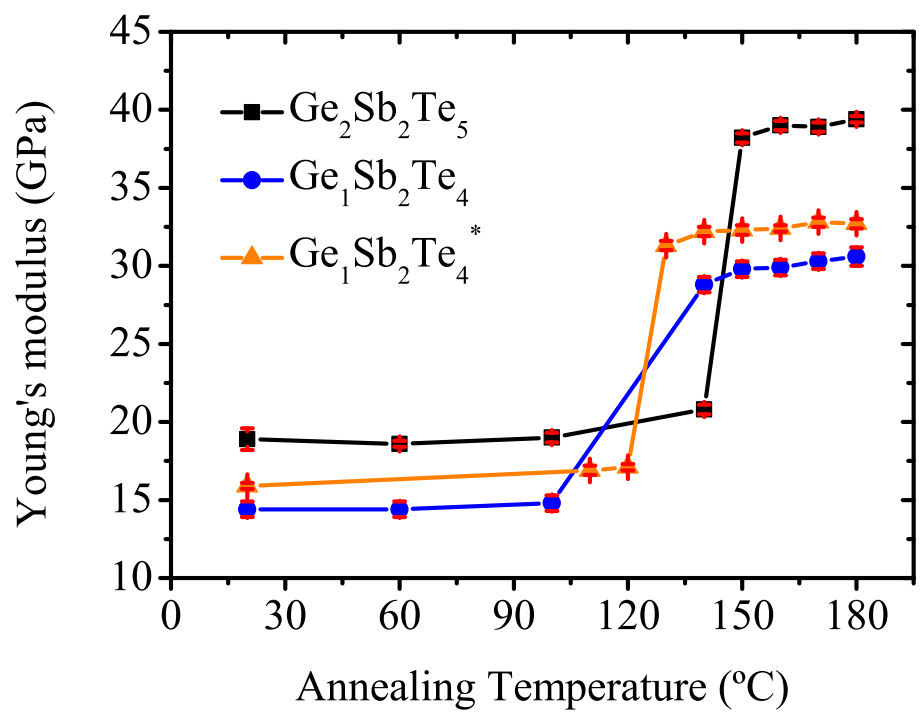

Figure 5: Young's modulus of the GST225 and GST124 thin films plotted as a function of the annealing temperature. We observe a rise in the Young's modulus value above the crystallization temperature for both the GST225 and GST124 thin films. The Young's modulus of the GST225 film is $30 \%$ higher than that of GST124. The GST124 composition marked with an asterix has a different anneal sequence, see table1. The lines are guides to the eye.

ria [15] and Kalb 34. The measured Young's moduli are lower than reported by Blachowicz et al. 28, who found 24.8 (0.06) GPa for the amorphous and 39.5 (0.8) GPa for the crystalline phase. In this work the Youngs modulus is derived from Brillouin light scattering experiments at phonon frequencies in the range of 2 to $16 \mathrm{GHz}$, so far above the resonance frequency of our cantilevers. Since the Youngs modulus in rigid materials is expected to increase slightly with frequency [35, this could very well explain the difference between both methods.

\subsection{Residual stress}

From the cantilever bending after annealing, the residual stress in the GST layers can be determined. This stress strongly depends on the annealing temperature as well as the material composition (see figure 6). We observed a 
sharp increase in the residual stress, when the films were annealed above the crystallization temperature.

There is no residual stress in the as-deposited GST225 thin film. This is agreement with work by Leervad-Pedersen et al 31, who demonstrate that stress is released in the amporphous state due to plastic flow. The stress increases slowly to a value of $49.7(0.3) \mathrm{MPa}$ at $140^{\circ} \mathrm{C}$, just before the crystallization temperature. A sharp increase in residual stress to a value of 331.6 (1.5) MPa was observed after annealing at $150^{\circ} \mathrm{C}$. The crystallization temperature determined from the stress measurements is consistent with the Young's modulus results. Annealing above the crystallization temperature up to $180^{\circ} \mathrm{C}$ shows some stress relaxation, see figure 6. The trend in the temperature dependence of the residual stress in GST225 agrees with results published by others [31, 36]. Krusin-Elbaum et al. observed a similar stress release, and showed it to be 255 dependant on the material composition [29].

Residual stress in the GST124 and GST124* thin films were also found to be negligible in the as-deposited state. The stress for GST124* increased to a value of $46.8 \mathrm{MPa}(0.2)$ at $120^{\circ} \mathrm{C}$. When measured after annealing at the crystallization temperature $\left(130^{\circ} \mathrm{C}\right)$, the residual stress increased to value of 238.6 (1.0) MPa. The GST124 and GST124* sample show distinct differences in residual stress after annealing above the crystallization temperature. The annealing history has a strong effect. The GST124* sample, which passed through more annealing steps (see table 1) shows a $45 \%$ higher residual stress than the GST124 sample at $140^{\circ} \mathrm{C}$. It is known that the crystallisation process is a function of the temperature as well as time [37. As the temperature ramp rate increases, the grainsize in the crystalline film decreases. It is not unlikely that this has a profound effect on the residual stress.

Unlike the GST225 thin film however, stress relaxation in the GST124 thin films at elevated annealing temperatures is not present. We suspect that this is 270 related to the fact that in GST225 has a meta-stable FCC crystal phase 26], which might be absent in GST124. 


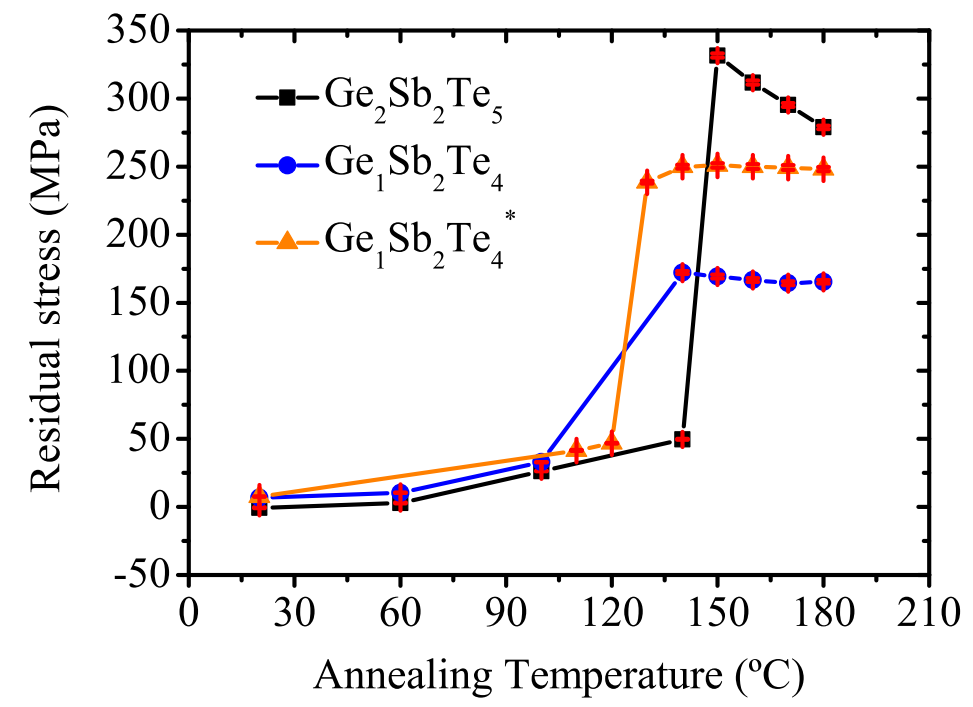

Figure 6: Residual stress dependence of $\mathrm{Ge}_{2} \mathrm{Sb}_{2} \mathrm{Te}_{5}$ and $\mathrm{Ge}_{1} \mathrm{Sb}_{2} \mathrm{Te}_{4}$ films on temperature. The $\mathrm{Ge}_{1} \mathrm{Sb}_{2} \mathrm{Te}_{4}$ films shows lower values of residual stress as compared to $\mathrm{Ge}_{2} \mathrm{Sb}_{2} \mathrm{Te}_{5}$. There is a clear difference in residual stress values for two identical $\mathrm{Ge}_{1} \mathrm{Sb}_{2} \mathrm{Te}_{4}$ samples, which were annealed through different steps (see table 1). Stress relaxation as seen in the $\mathrm{Ge}_{2} \mathrm{Sb}_{2} \mathrm{Te}_{5}$ film after $150^{\circ} \mathrm{C}$ was not observed for $\mathrm{Ge}_{1} \mathrm{Sb}_{2} \mathrm{Te}_{4}$ films. The lines are guides to the eye. 


\subsection{Crystallization temperature}

The variation in the Young's modulus and residual stress of the GST225 thin films with annealing temperature is compared with changes in the trans-

275 transimissivity coincides well with the rise of the Young's modulus and residual stress. This crystallization temperature of $150^{\circ} \mathrm{C}$ film agrees as well with data published for $\mathrm{Ge}_{2} \mathrm{Sb}_{2} \mathrm{Te}_{5}$ in [32, 39. Therefore the observed changes in mechancal properties have the same origin as the change in transmissivity, and

\subsection{Strain}

From the measured residual stress and the Young's modulus at room temperature, we can calculate the strain in the film. The result is plotted in figure 8 The drop in the residual stress of the GST225 thin film after the crystallization temperature (figure 6) is also reflected in a steep drop in the strain values. Likewise, the strain in the GST124 thin films follows the stress behaviour after the crystallization temperature, because of the negligible variation in the Young's modulus.

\subsection{Sheet resistance}

Next to change in Youngs modules, increase in stress and reduction of transmissivity, the crystallisation of the GST layers also leads to a change in electrical resistance. Therefore the sheet resistance was measured as a function of the annealing temperature, see figure 9 The sheet resistance of both the GST225 and GST124 thin films in the as-deposited amorphous state is high (in the range of 1100 to $1200 \mathrm{k} \Omega / \mathrm{sq}$ ) compared to the crystalline state. Annealing with increasing temperature reduces the sheet resistance monotonously. This is in contrast with the sudden changes in mechanical properties, and with previously reported work. Siegrist [40], Jang et al. [41] and Njoroge et al. 26] for instance observed a sudden drop in resistance around the annealing temperature. 


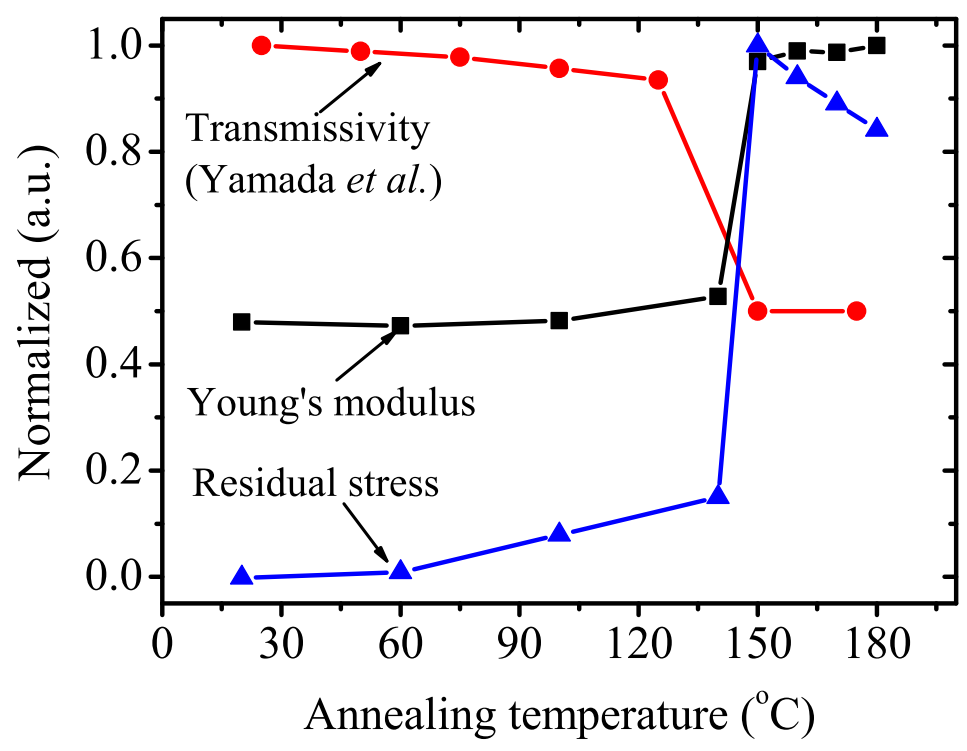

Figure 7: Young's modulus and residual stress of the GST225 thin film compared to the change in transmissivity at the crystallization temperature [38. The lines are guides to the eye. 


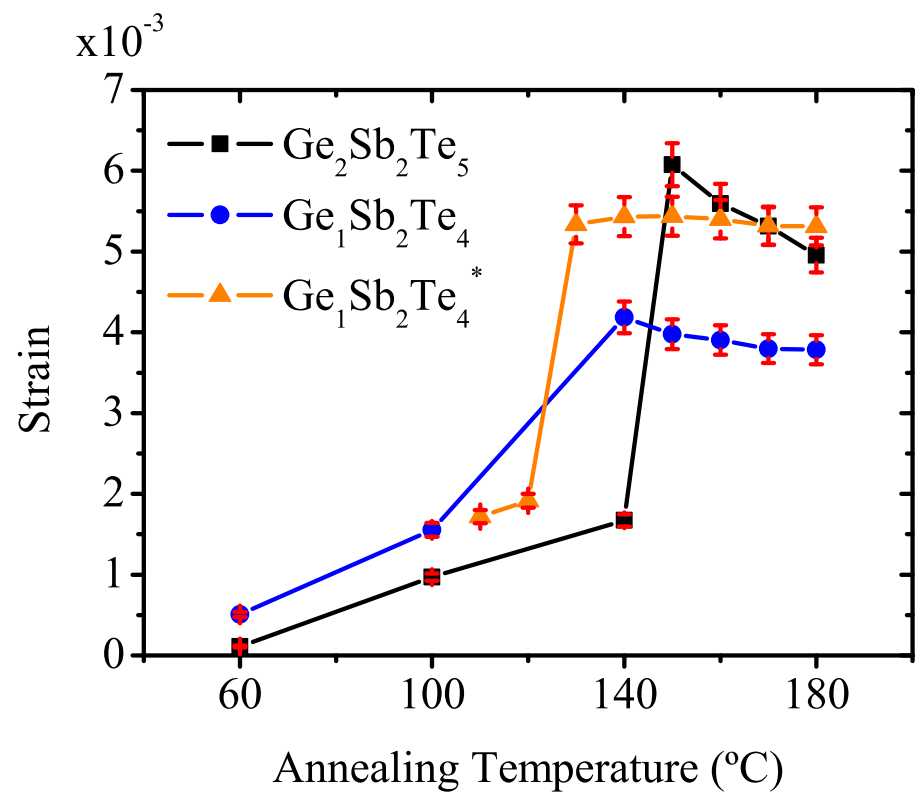

Figure 8: Strain in the GST thin films as a function of annealing temperature. The strain is estimated from the measured Young's modulus and residual stress at the corresponding annealing temperature. 


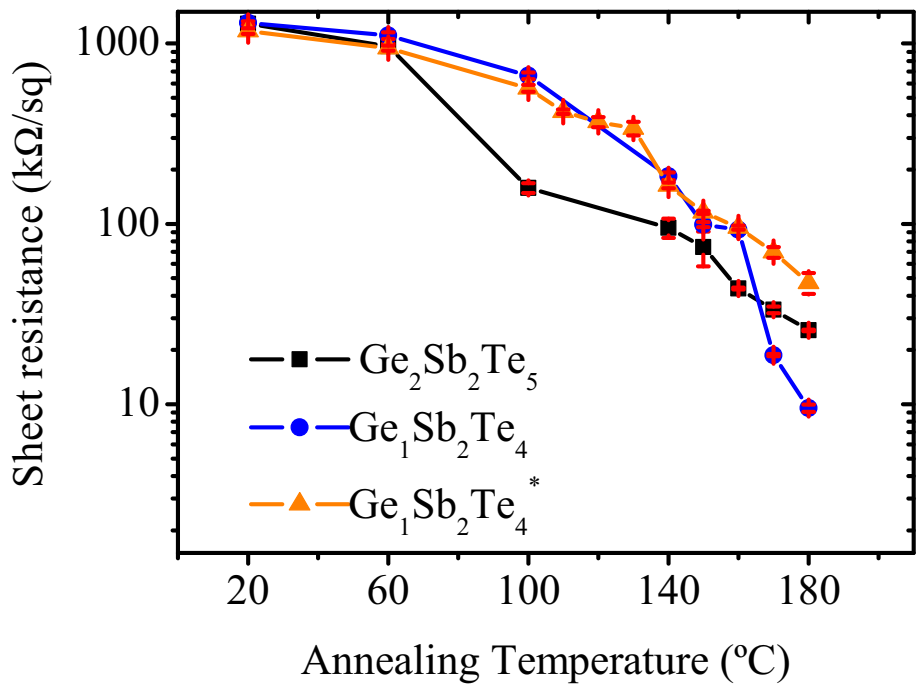

Figure 9: Change in the sheet resistance of the GST225 and GST124 films as a function of temperature. The sheet resistance was measured using a four-point probe at room temperature. The lines are guides to the eye.

At a the crystallisation temperature of $150^{\circ} \mathrm{C}$, the sheet resistance was $74 \mathrm{k} \Omega / \mathrm{sq}$ for the GST225 thin film, which is in the same order of magnitude as reported in literature. However, the sheet resistance in amorphous state is one order of magnitude less than reported values in literature $\left(\sim 10^{7} \mathrm{ohm} / \mathrm{sq}\right)$. We suspect that these lower values, and the absence of a sudden drop, are caused by the silicon device layer underneath the GST thin films. A shortcut current through this layer might reduce the sheets resistance and obscure a sudden drop at the crystallisation temperature. The effect of the resistance measurement itself on the phase change transition should however also be considered [42].

The sheet resistance of the GST124 and GST124* samples are generally 310 higher than the GST225 film. At $140^{\circ} \mathrm{C}$ was found to be $184 \mathrm{k} \Omega / \mathrm{sq}$ and $164 \mathrm{k} \Omega / \mathrm{sq}$ respectively (see figure 9 ). Above $150^{\circ} \mathrm{C}$ however, we again observe a clear dependence on annealing history. 


\section{Conclusion}

We have deposited amorphous $200 \mathrm{~nm}$ GST films on $3 \mu \mathrm{m}$ thick, 250 to

315 perature to induce a phase change. After annealing, the cantilever resonance frequency shifts up by approximately $500 \mathrm{~Hz}$ and the cantilevers bend upwards by about $6 \mu \mathrm{m}$.

From these changes in resonance frequency and radius of bending curvature ing from the amorphous to crystalline state. Calculations show that the effect of a change in neutral line when the thickness of the GST film changes by $6.5 \%$ is negligble. Therefore, we can consider the product of GST Young's modulus and film thickness, as well as the product of residual stress and film thickness, process.

We investigate two compositions, $\mathrm{Ge}_{1} \mathrm{Sb}_{2} \mathrm{Te}_{4}$ (GST124) and $\mathrm{Ge}_{2} \mathrm{Sb}_{2} \mathrm{Te}_{5}$ (GST225). The Young's modulus increases sharply from 18.9 (0.7) GPa to 38.2 (0.3) GPa (GST225) and 15.9 (0.2) GPa to 31.3 (0.3) GPa (GST124) after annealing above $\left(150^{\circ} \mathrm{C}\right)$ was slightly higher than that of GST124 $\left(130^{\circ} \mathrm{C}\right)$. Both values agree well with values quoted in literature obtained by optical reflection [38] and electrical conductivity [26].

Residual stress in the GST thin films increases sharply from almost no stress 335 $\left(\mathrm{GST}_{124}{ }^{*}\right)$ when changing from the amorphous to crystalline phase. The increase of stress follows the same temperature behaviour as measured for the Young's modulus.

We observed relaxation in the residual stress of the GST225 thin film when annealed above the crystallization temperature. This relaxation is not present in the GST124 films. The residual stress is highly dependent on the annealing history, we observed higher stress values if the film is annealed longer below the 
crystallization temperature.

The sheet resistance measured for the two compositions of GST shows one order of magnitude difference between the amorphous and crystalline state. Unlike the Young's modulus and residual stress, there is no sharp transition temperature. The resistance rather drops monotonously over a wide temperature range. This might be caused by the fact that we measure the sheet resistance of the phase change material directly on the semiconducting silicon substrate.

The cantilever based method analysis method presented here provides valuable information on the mechanical properties of GST124 and GST224 phase change films. It can be easily extended to films of different thicknesses and composition. Moreover, we demonstrate that phase change materials allow for significant changes in resonance frequency and curvature of micro-cantilevers.

355 Since crystallisation of phase change films is in principle reversible, we believe they can be succesfully applied as actuator material in low-power micromechanical devices. We envision tuneable resonantors that do not require energy after tuning the resonance frequencies, or switches that only require power during a change in position.

\section{Acknowledgements}

The authors are indebted to Andrew Pauza of Plarion inc. for the deposition of the GST film, Meint de Boer for etching, Remco (Pino) Sanders for laser Doppler vibrometer measurements, Johnny Sanderink and Henk van Wolferen for SEM. The authors would like to thank dr. Niels Tas and prof. Miko Elwenspoek of the University of Twente, prof. Matthias Wuttig of the RWTH Aachen and prof. David Wright of Exeter University for fruitful discussion.

The authors gratefully acknowledge the support of the SmartMix Program (SmartPie) of the Netherlands Ministry of Economic Affairs and the Netherlands Ministry of Education, Culture and Science. HB is grateful to EPSRC for funding via grants EP/J00541X/2 and EP/J018694/1 


\section{References}

[1] P. Zhao, Z. Zhao, D. Hunter, R. Suchoski, C. Gao, S. Mathews, M. Wuttig,

I. Takeuchi, Fabrication and characterization of all-thin-film magnetoelectric sensors, Applied Physics Letters 94 (24) (2009) 243507.

375 ـ 2] S. Dong, J. Zhai, F. Bai, J.-F. Li, D. Viehland, Push-pull mode magne-

प tostrictive/piezoelectric laminate composite with an enhanced magnetoelectric voltage coefficient, Applied Physics Letters 87 (6) (2005) 062502.

[3] H. Bhaskaran, M. Li, D. Garcia-Sanchez, P. Zhao, I. Takeuchi, H. X. Tang, Active microcantilevers based on piezoresistive ferromagnetic thin films, Appl. Phys. Lett. 98 (1) (2011) 013502.

[ [4] A. Sebastian, N. Papandreou, A. Pantazi, H. Pozidis, E. Eleftheriou, Nonresistance-based cell-state metric for phase-change memory, J. Appl. Phys. 110 (8) (2011) 084505.

[ [5] W. Pernice, H. Bhaskaran, Photonic non-volatile memories using phase change materials, Applied Physics Letters 101 (17) (2012) 171101.

[ [6] C. Rios, P. Hosseini, C. Wright, H. Bhaskaran, W. Pernice, On-chip photonic memory elements employing phase-change materials, Advanced Materials 26 (9) (2014) 1372-1377.

q [7] P. Hosseini, C. Wright, H. Bhaskaran, An optoelectronic framework enabled by low-dimensional phase-change films, Nature 511 (7508) (2014) 206-211.

[8] R. H. Poelma, H. Sadeghian, S. P. M. Noijen, J. J. M. Zaal, G. Q. Zhang, A numerical experimental approach for characterizing the elastic properties of thin films: Application of nanocantilevers, J. Micromech. Microeng. 21 (6) (2011) 065003.

395 [9] D. Isarakorn, A. Sambri, P. Janphuang, D. Briand, S. Gariglio, J.-M. Triscone, F. Guy, J. W. Reiner, C. H. Ahn, N. F. de Rooij, Epitaxial 
piezoelectric MEMS on silicon, J. Micromech. Microeng. 20 (5) (2010) art. no. 055008.

[10] H. Ræder, F. Tyholdt, W. Booij, F. Calame, N. P. Østbø, R. Bredesen, K. Prume, G. Rijnders, P. Muralt, Taking piezoelectric microsystems from the laboratory to production, J. Electroceram. 19 (4) (2007) 357-362.

[11] H. Nazeer, M. D. Nguyen, L. A. Woldering, L. Abelmann, G. Rijnders, M. C. Elwenspoek, Determination of the Young's modulus of pulsed laser deposited epitaxial PZT thin films, J. Micromech. Microeng. 21 (7) (2011) 074008 .

[12] H. Nazeer, L. A. Woldering, L. Abelmann, M. D. Nguyen, G. Rijnders, M. C. Elwenspoek, Influence of silicon orientation and cantilever undercut on the determination of the Young's modulus of thin films, Microelectron. Eng. 88 (8) (2011) 2345-2348.

${ }_{410}$ [13] Y. Won, J. Lee, M. Asheghi, T. Kenny, K. Goodson, Phase and thickness dependent modulus of $\mathrm{Ge}_{2} \mathrm{Sb}_{2} \mathrm{Te}_{5}$ films down to $25 \mathrm{~nm}$ thickness, Applied Physics Letters 100 (16) (2012) 161905.

[14] H. Nazeer, M. D. Nguyen, A. J. H. M. Rijnders, L. Abelmann, M. C. Elwenspoek, Compositional dependence of the young's modulus and piezoelectric coefficient of (110)-oriented pulsed laser deposited pzt thin films, Journal of microelectromechanical systems 24 (1) (2014) 166-173.

[15] E. Carria, A. M. Mio, S. Gibilisco, M. Miritello, M. G. Grimaldi, E. Rimini, Tuning the crystallization temperature of amorphous $\mathrm{Ge}_{2} \mathrm{Sb}_{2} \mathrm{Te}_{5}$ by $\mathrm{O}$ and Si recoil implantation, Electrochem. Solid-State Lett. 14 (3) (2011) H124H127.

[16] J. Tomforde, W. Bensch, L. Kienle, V. Duppel, P. Merkelbach, M. Wuttig, Thin films of Ge-Sb-Te-based phase change materials: Microstructure and in situ transformation, Chem. Mater. 23 (17) (2011) 3871-3878. 
[17] M. Krbal, A. V. Kolobov, J. Haines, A. Pradel, M. Ribes, P. Fons, J. Tominaga, C. Levelut, R. Le Parc, M. Hanfland, Temperature independence of pressure-induced amorphization of the phase-change memory alloy $\mathrm{Ge}_{2} \mathrm{Sb}_{2} \mathrm{Te}_{5}$, Appl. Phys. Lett. 93 (3) (2008) 031918.

[18] T. Matsunaga, N. Yamada, Structural investigation of $\mathrm{GeSb}_{2} \mathrm{Te}_{4}$ : A high-speed phase-change material, Phys. Rev. B 69 (10) (2004) 10411111041118.

[19] A. Marmier, K. Kohary, C. D. Wright, Determination of the anisotropic elastic properties of $\mathrm{Ge}_{1} \mathrm{Sb}_{2} \mathrm{Te}_{4}$, Appl. Phys. Lett. 98 (23) (2011) 231911.

[20] C.-A. Jong, W. Fang, C.-M. Lee, T.-S. Chin, Mechanical properties of phase-change recording media: GeSbTe films, Jpn. J. Appl. Phys. 40 (5 A) (2001) 3320-3325.

[21] V. Weidenhof, I. Friedrich, S. Ziegler, M. Wuttig, Atomic force microscopy study of laser induced phase transitions in $\mathrm{Ge}_{2} \mathrm{Sb}_{2} \mathrm{Te}_{5}$, J. Appl. Phys. 86 (10) (1999) 5879-5887.

[22] J.-A. Schweitz, A new and simple micromechanical approach to the stressstrain characterization of thin coatings, J. Micromech. Microeng. 1 (1) (1991) 10-15.

[23] G. Stoney, The tension of metallic films deposited by electrolysis, Proc. R. Soc. Lond. A 82 (553) (1909) 172-175.

[24] S. Jeon, T. Thundat, Instant curvature measurement for microcantilever sensors, Appl. Phys. Lett. 85 (6) (2004) 1083-1084.

[25] D. Weaire, M. F. Ashby, J. Logan, M. J. Weins, On the use of pair potentials to calculate the properties of amorphous metals, Acta Metallurgica 19 (8) (1971) 779-788.

[26] W. K. Njoroge, H.-W. Wöltgens, M. Wuttig, Density changes upon crys450 tallization of $\mathrm{Ge}_{2} \mathrm{Sb}_{2.04} \mathrm{Te}_{4.74}$ films, J. Vac. Sci. Technol. A 20 (1) (2002) 230-233. 
[27] S. Elliott, J. Hegedus, Computer simulation of the phase-change cycle of GST-225, in: MRS Proceedings, 1072 , 1072-G01-02, Vol. 1072, San Francisco, CA, 2008, pp. 99-107.

[28] T. Blachowicz, M. G. Beghi, G. Güntherodt, B. Beschoten, H. Dieker, M. Wuttig, Crystalline phases in the $\mathrm{GeSb}_{2} \mathrm{Te}_{4}$ alloy system: Phase transitions and elastic properties, J. Appl. Phys. 102 (9) (2007) 093519.

[29] L. Krusin-Elbaum, C. Cabral, K. N. Chen, M. Copel, D. W. Abraham, K. B. Reuter, S. M. Rossnagel, J. Bruley, V. R. Deline, Evidence for segregation of $\mathrm{Te}$ in $\mathrm{Ge}_{2} \mathrm{Sb}_{2} \mathrm{Te}_{5}$ films: Effect on the "phase-change" stress, Appl. Phys. Lett. 90 (14) (2007) 141902.

[30] J. Kalb, F. Spaepen, M. Wuttig, Calorimetric measurements of phase transformations in thin films of amorphous Te alloys used for optical data storage, J. Appl. Phys. 93 (5) (2003) 2389-2393.

[31] T. P. Leervad Pedersen, J. Kalb, W. K. Njoroge, D. Wamwangi, M. Wuttig, F. Spaepen, Mechanical stresses upon crystallization in phase change materials, Appl. Phys. Lett. 79 (22) (2001) 3597-3599.

[32] J. Kalb, F. Spaepen, T. P. Leervad Pedersen, M. Wuttig, Viscosity and elastic constants of thin films of amorphous te alloys used for optical data storage, J. Appl. Phys. 94 (8) (2003) 4908-4912.

[33] B. Kalkan, S. Sen, B. G. Aitken, S. V. Raju, S. M. Clark, Negative P-T slopes characterize phase change processes: Case of the $\mathrm{Ge}_{1} \mathrm{Sb}_{2} \mathrm{Te}_{4}$ phase change alloy, Phys. Rev. B 84 (1) (2011) 014202.

[34] J. A. Kalb, M. Wuttig, F. Spaepen, Calorimetric measurements of structural relaxation and glass transition temperatures in sputtered films of amorphous Te alloys used for phase change recording, J. Mater. Res. 22 (3) (2007) 748-754. 
[35] T. Pritz, Frequency dependences of complex moduli and complex poisson's ratio of real solid materials, Journal of Sound and Vibration 214 (1) (1998) 83-104.

[36] I.-M. Park, J.-K. Jung, S.-O. Ryu, K.-J. Choi, B.-G. Yu, Y.-B. Park, S. M. Han, Y.-C. Joo, Thermomechanical properties and mechanical stresses of $\mathrm{Ge}_{2} \mathrm{Sb}_{2} \mathrm{Te}_{5}$ films in phase-change random access memory, Thin Solid Films 517 (2) (2008) 848-852.

[37] G. Burr, P. Tchoulfian, T. Topuria, C. Nyffeler, K. Virwani, A. Padilla, R. Shelby, M. Eskandari, B. Jackson, B.-S. Lee, Observation and modeling of polycrystalline grain formation in $\mathrm{Ge}_{2} \mathrm{Sb}_{2} \mathrm{Te}_{5}$, Journal of Applied Physics 111 (10) (2012) 104308.

[38] N. Yamada, E. Ohno, K. Nishiuchi, N. Akahira, M. Takao, Rapid-phase transitions of $\mathrm{GeTe}-\mathrm{Sb}_{2} \mathrm{Te}_{3}$ pseudobinary amorphous thin films for an optical disk memory, J. Appl. Phys. 69 (5) (1991) 2849-2856.

[39] I. Friedrich, V. Weidenhof, W. Njoroge, P. Franz, M. Wuttig, Structural transformations of $\mathrm{Ge}_{2} \mathrm{Sb}_{2} \mathrm{Te}_{5}$ films studied by electrical resistance measurements, J. Appl. Phys. 87 (9 I) (2000) 4130-4134.

[40] T. Siegrist, P. Jost, H. Volker, M. Woda, P. Merkelbach, C. Schlockermann,

n. Muttig, Disorder-induced localization in crystalline phase-change materials, Nature Materials 10 (3) (2011) 202-208.

[41] M. H. Jang, S. J. Park, D. H. Lim, M.-H. Cho, Y. K. Kim, H.-J. Yi, H. S. Kim, Structural stability and phase-change characteristics of $\mathrm{Ge}_{2} \mathrm{Sb}_{2} \mathrm{Te}_{5} / \mathrm{SiO}_{2}$ nano-multilayered films, Electrochem. Solid State Letters 12 (4) (2009) H151-H154.

[42] Z.-Q. Cheng, G.-G.and Zhang, J.-N. Ding, Z. Ling, Y.-B. Chen, Mechanical and electrical properties of gesb2te4 film with external voltage applied, Applied Surface Science 285 (PARTB) (2013) 532-537. 
[43] H. V. Jansen, M. J. De Boer, S. Unnikrishnan, M. C. Louwerse, M. C. Elwenspoek, Black silicon method X: a review on high speed and selective plasma etching of silicon with profile control: an in-depth comparison between Bosch and cryostat DRIE processes as a roadmap to next generation equipment, Journal of Micromechanics and Microengineering 19 (3) (2009) 033001 .

[44] R. A. Brookhuis, T. S. J. Lammerink, R. J. Wiegerink, M. J. De Boer, M. C. Elwenspoek, Force sensor for measuring power transfer between the human body and the environment, in: Proc. $16^{\text {th }}$ International Solid-State Sensors, Actuators and Microsystems Conference, TRANSDUCERS'11, Beijing, 2011, pp. 2042-2045.

\section{Appendix}

\subsection{Fabrication process}

Figure 10 shows the fabrication scheme for the cantilevers using a dedicated SOI/MEMS fabrication process. The process is described briefly as follows. (a) A double side polished silicon on insulator (SOI) wafer was selected. The substrate has a $380 \mu \mathrm{m}$ handle wafer and a $3 \mu \mathrm{m}$ thick device layer. The device layer defines the thickness of the cantilevers. A layer of $500 \mathrm{~nm}$ buried oxide (BOX) serves as an etch stop during the etching of the device layer and handle wafer. A photoresist mask was designed with cantilevers that have varying lengths from $250 \mu \mathrm{m}$ to $350 \mu \mathrm{m}$ in steps of $10 \mu \mathrm{m}$. The cantilevers have a fixed width of $30 \mu \mathrm{m}$. ( $b$ and $c$ ) The front side of the (001) single crystal silicon device layers was patterned by conventional UV photolithography to define the shape of the cantilevers. The photoresist used was Olin907-17 with a thickness of $1.7 \mu \mathrm{m}$. (d) Subsequently the cantilevers were anisotropically etched by deep 530 reactive ion etching (DRIE) using $\mathrm{SF}_{6}, \mathrm{O}_{2}$ and $\mathrm{C}_{4} \mathrm{~F}_{8}$ gases [43. (e and $f$ ) $\mathrm{A}$ layer of $3.5 \mu \mathrm{m}$ thick photoresist (908-35) was applied on the back side of the wafer and patterned to define holes for cantilevers release. $(g)$ DuPont MX-5020 


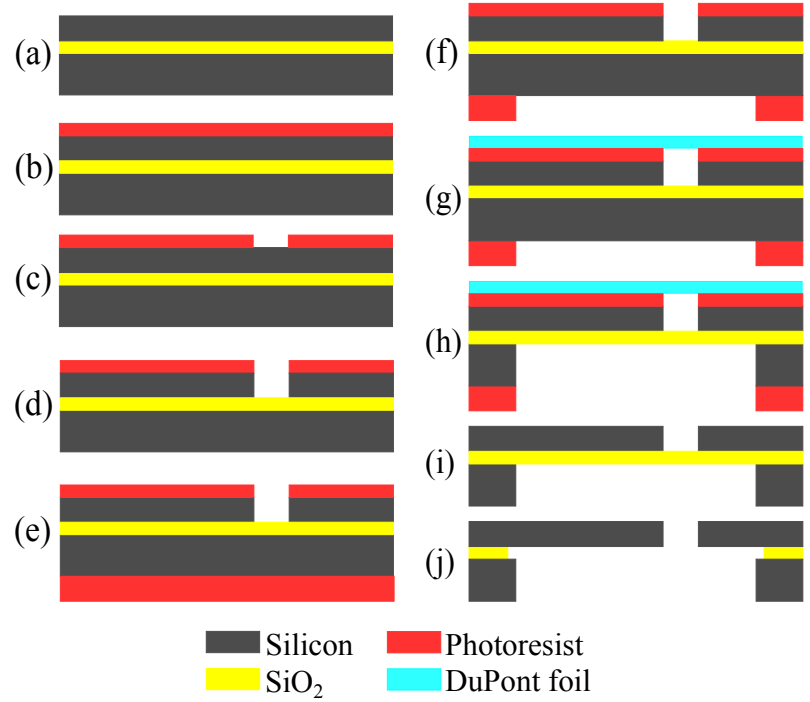

Figure 10: Fabrication process of the silicon cantilevers. (a) A SOI wafer with a $3 \mu \mathrm{m}$ thick device layer and a $500 \mathrm{~nm}$ thick BOX was selected. (b and c) Application and patterning of the photoresist (Olin 907-17) on the front side of the wafer. (d) Silicon device layer was etched by DRIE. (e and f) Thick photoresist (Olin 908-35) was applied and patterned on the back side of the wafer. (g) Application of the foil (DuPont MX5020) on the front side for stable temperature control and avoid helium leakage. (h) Through holes from the back side were etched by DRIE. (i) Photoresist and foil was removed from the front and back side using oxygen plasma. (j) Cantilevers were released by etching the BOX layer using BHF.

foil was applied on the front side of the wafers to protect the cantilevers from damage and prevent leakage of the helium during the wafer through etching. Application of this foil was required to ensure stable temperature control of the wafer during the back side DRIE process [44]. (h) Etching of the handle wafer from the back side was then performed by DRIE using $\mathrm{SF}_{6}, \mathrm{O}_{2}$ and $\mathrm{C}_{4} \mathrm{~F}_{8}$ gases [43]. 